\title{
1 \\ Introduction to Fragment-based Drug Discovery
}

\author{
Mike Cherry and Tim Mitchell
}

\subsection{Introduction}

Fragment screening is the process of identifying relatively simple, often weakly potent, bioactive molecules. It is gaining wide acceptance as a successful hit-finding technique both in its own right and as a method of finding hit molecules when traditional high-throughput screening (HTS) methods fail. Fragment hits are typically highly 'ligand efficient', i.e. possess a high binding affinity per heavy atom, and thus are ideal for optimisation into clinical candidates with good drug-like properties. Fragment screening is being increasingly proven as a successful means of generating novel chemical starting material for drug discovery programmes. It has been the subject of numerous publications and reviews in the last few years. ${ }^{[1-5]}$

Fragment screening was initially developed to generate hit compounds against targets for which other methods, such as HTS, had been unsuccessful. ${ }^{[6]}$ At the same time, many shortcomings of HTS were becoming increasingly apparent:

1. The hits being generated from high-throughput screening of combinatorial chemistryderived libraries are not particularly suitable for lead optimisation programmes: These compounds tended to be large and hydrophobic and thus had limited potential for development before becoming in violation of 'drug-like' parameters as described by Lipinski et $a l .{ }^{[7]}$ 'Garbage in, garbage out really applies to drug screening', as Lipinski et al. quote. $^{[8]}$ 
2. Hit rates from HTS are often low and the hits obtained fail to progress into lead optimisation: HTS is the predominant technique for hit finding employed by the majority of pharmaceutical companies and is central to modern drug discovery. However, many scientists are now regarding HTS as a costly necessity rather than a method of choice. ${ }^{[9]}$

3. HTS only samples a minute fraction of drug-like chemical space: A widely quoted estimate $^{[10]}$ of the number of molecules containing up to $30 \mathrm{C}, \mathrm{N}, \mathrm{O}$ or S atoms exceeds $10^{60}$ (and the mass of a 1 pmol sample of each would of the same order as that of the observable universe). An HTS screen of, say, $10^{6}$ molecules would only sample a minute portion of this space. By contrast, the number of synthetically feasible small molecule fragments with masses up to $160 \mathrm{Da}$ has been estimated ${ }^{[11]}$ at $10^{7}$, hence a typical fragment screen of $10^{3}-10^{4}$ molecules is sampling a much higher proportion of this space.

4. Many companies have realised, to their significant cost, that screening non-proprietary vendor compounds against non-proprietary targets can lead to difficulties in securing a good intellectual property (IP) position: Increasingly, there is the possibility that another company has screened similar compounds against a similar target and therefore it is difficult to obtain strong patent protection against the chemical series. If competitor patents do not appear for some time after the initiation of a drug discovery programme, significant resources can be wasted on attempting to optimise compounds that another organisation had already discovered and patented ${ }^{[12]}$.

Hence there is increased pressure to discover more suitable chemical starting points and the term 'lead-like' has been used to describe these less complex molecules. ${ }^{[13]}$ By its very nature, fragment screening is ideally suited to finding low molecular weight bioactive compounds. Because these compounds tend to be relatively low in potency (typically in the $100-1000 \mu \mathrm{M}$ range) they are not identifiable by an HTS run at a typical compound concentration of $10-30 \mu \mathrm{M}$. Fragment screening collections, even those formed from non-proprietary vendor compound collections, lie beyond the scope of HTS compound collections, thus increasing the chances of identifying novel molecules that can be optimised into patentable series.

Subsequent chapters will discuss in more detail and provide case studies of best practices of using fragments in drug discovery programmes. It is useful, though, to start with an understanding of what is meant by a fragment and how the use of fragments has reached the current level of popularity.

\subsection{What is a Fragment?}

A quick search of the literature using the term 'drug-likeness' throws up thousands of articles, reflecting the aspiration of the industry to be able to classify compounds readily as drug-like or not. A wide variety of methodologies has been employed in the process of classification, from simple filters based on physicochemical descriptors through to more complex QSAR models. Although the concept of fragment-based drug discovery has been around since the early 1980 s, the application is relatively new and as such the volume of literature around the subject can be measured in hundreds, not thousands. There are 
a rapidly increasing number of studies investigating what makes a good starting point in fragment-based drug discovery and how to formulate libraries to maximize success in the screening process.

The exact nature of a fragment library is very much dependent on the screening protocol; however, the methods employed to construct fragment libraries borrow heavily from experiences in drug-like classification. A simple analogy is that of the Astex rule-ofthree ${ }^{[14]}$ as compared with Lipinski et al.'s 'rule-of-five ${ }^{\text {,[15] }}$. Here the same physicochemical descriptors are used but compounds are limited to a molecular weight (MW) of $300 \mathrm{Da}$, three or less hydrogen bond donors and acceptors and a calculated $\log P$ of $\leq 3$. The Astex library is comprised of several hundred small organic ring systems, is a mixture of target-specific and general-purpose fragments and is used to probe a target site using high-throughput $\mathrm{X}$-ray crystallographic screening.

The application of a filter such as the 'rule-of-three' and subsequent identification of target-specific compounds are in general the penultimate steps in the virtual selection of fragments from a significantly larger chemical space. Additional steps common to most, if not all, selection criteria for fragment libraries, particularly if starting from commercial vendor space, include the removal of undesirable chemical functionality, elimination of poorly soluble compounds, a selection based on synthetic tractability and consideration of scaffold diversity. It is also probably safe to say that the final step in most virtual screening campaigns involves scientists eyeballing compounds, predominantly to make the final selection but also to ensure the baby is not being thrown out with the bath water. The virtual screening process is commutative with respect to the final result, excepting the manual selection; however, the order of operation will impact on efficiency. 1D and 2D filters, such as the exclusion of undesirable chemical functionality using substructure searching, can eliminate a high percentage of compounds, ${ }^{[16]}$ thus reducing the resources needed for computationally more expensive procedures such as pharmacophore searching and high-throughput docking.

$\mathrm{SGX}^{[17]}$ outline a series of such criteria in the selection of a $\sim 1000$ member diverse fragment library that includes filters for $\mathrm{MW}, C \log P$, compound complexity, exclusion of undesirable chemical functionality, solubility, ring system diversity, synthetic accessibility and, interestingly, a selection based on bromination. All the compounds in the library have $\leq 16$ heavy atoms, $\geq 1$ ring and at least two synthetic handles. Most of the compounds obey the 'rule-of-three' and, importantly for screening using X-ray crystallography, $60 \%$ have high solubility. Whereas Astex have target-specific fragments, SGX make no such distinction on the basis that hit rates are in general higher in fragment screening and a library of 1000 diverse fragments is deemed large enough to yield sufficient chemical matter to initiate a discovery programme. The size of a fragment library, the complexity of the molecules within the library and the optimisability are all characteristics important to fragment screening and are discussed in more detail below.

Approximately half of the compounds in the SGX library contain bromine, a feature included to enhance synthetic elaboration but also aid in the detection and validation of crystallographic screening data. Compounds in the original 'SHAPES' library developed at Vertex, ${ }^{[18]}$ in addition to many of the aforementioned filters, had to yield simple ${ }^{1} \mathrm{H}$ NMR spectra and contain at least two protons within $5 \AA$ of one another, both aids to the screening of mixtures using nuclear magnetic resonance (NMR) techniques. The library stemmed from previous work investigating the properties of known drugs, ${ }^{[19,20]}$ where 
computational methods were used to break down and analyse the constituent components of a database of commercially available drug molecules. Molecules are split into ring systems, linkers, side-chains and frameworks, where a framework is defined as the union of ring systems and linkers in a molecule. A surprisingly small number of frameworks (41), taking into consideration atom types and bond orders, describe the framework of $\sim 24 \%$ of the molecules in the database. These frameworks, along with 30 of the most common side-chains, were used in the process of selecting compounds from the ACD (MDL Available Chemicals Directory) for inclusion in the SHAPES library. The final library contained commercially available compounds that are water soluble at $1 \mathrm{mM}$, have MW in the range 68-341 Da (average $194 \mathrm{Da}$ ), 6-22 heavy atoms and a $C \log P$ of -2.2 to 5.5. The library profile reflects the fact that the design was dominated by the selection of suitable frameworks and side-chains and the requirement for high aqueous solubility. More recent design strategies incorporate physicochemical property filters where it would be unusual to pass compounds with such high $\log P$ values.

Breaking down drug-like molecules into fragments would at face value seem an obvious starting point for a fragment library. One of the issues, however, associated with earlier attempts to use fragments was that chemistry featured poorly in the fragmentation process, leading to synthetic difficulties in subsequent application. Consequently, the breakdown of drug-like compounds into fragments has been automated in computational methods, such as RECAP, ${ }^{[21]}$ that are chemically intuitive. Originally cited as a means of identifying privileged molecular building blocks for constructing combinatorial libraries rich in biological motifs, these methods are equally suited to generating libraries of fragments for screening and ensuing optimisation studies. Synthetic optimisability features heavily in Novartis's design for a fragment library. First an analysis was undertaken looking at results from NMR screening as compared with HTS results in relation to the Hann complexity model ${ }^{[22]}$ The analysis, which is discussed in more detail below, supports the basic principles of fragmentbased discovery ${ }^{[23]}$ and provided the framework from which to design a next-generation fragment library. Emphasis is placed on the ability to optimise low-affinity hits through incorporating one or more synthetic handles. Investigation of previous attempts to utilize synthetically tractable functionality in fragments highlighted the fact that in these smaller, less complex molecules the functional group is more often than not an integral component in binding to the target protein. Strategies for increasing the likelihood that a synthetic handle is available for modification include masking the functionality, selection of functionality that is normally not recognised by a protein or simply incorporating multiple groups. Novartis employed what they term a fragment pair strategy, where a fragment building block with an exposed synthetic handle is transformed into a screening fragment by masking the functional group but having minimal effect on a range of computed properties. The fragment building block can then be used in subsequent elaboration when a hit is observed for the corresponding fragment screening compound. In addition to library profiles akin to those outlined above, Similog keys are used as a measure of complexity. Similog keys represent pharmacophoric triplets and it was found that, as expected, fragments with micromolar to millimolar potency are significantly lower in complexity than the more drug-like molecules used in HTS.

Vernalis also use NMR screening in their strategy for fragment-based drug discovery called SeeDs ${ }^{[24]}$ (Selection of Experimentally Exploitable Drug start points). A pharmacophore fingerprint is used as a measure of chemical complexity and diversity amongst 
compounds as part of the virtual selection, to give a fragment library that has evolved over several generations. The fingerprints, which are essentially identical in nature to Similog keys noted above, encode the presence of pharmacophoric triangles comprised of standard features such as hydrogen bond donors/acceptors, hydrophobes and aromatics. The more pharmacophoric triangles in a molecule, the longer is the fingerprint, the length of which is taken to represent the complexity of the molecule. Within the context of the library as a whole, the fragment fingerprints are compared with fingerprints calculated for a drug-like and a protein binding set of reference compounds. The comparison is made at increasing limits in the distance between features in a pharmacophoric triangle, thus functioning as a rough guide to the diversity of the library with increasing molecular size. It also allows the selection of compounds that are novel to either of the reference sets. Novelty, as previously mentioned, is another advantage of fragment-based discovery as it operates in chemical space not normally identified by HTS. The physicochemical profile of the library is very similar to those outlined above; most (99\%) of the fragments in the 1300 library have MW $\leq 300, S \log P \leq 3$ and $\leq 3$ hydrogen bond donors, $90 \% \leq 3$ hydrogen bond acceptors and $\sim 80 \% \leq 3$ rotatable bonds and polar surface area (PSA) $\leq 60 \AA^{2}$.

The SeeDs strategy uses NMR experiments to identify fragments that bind competitively to a specific site of the target protein and then X-ray crystallography to determine the exact pose of a fragment hit. SGX also advocates the combination of methodologies using a high-concentration biochemical assay in conjunction with X-ray crystallography. The combination of approaches helps to circumvent the individual shortcomings of each method if used on an individual basis. For instance, false positives are inevitable when screening at high concentrations in a biochemical assay. X-ray crystallography therefore provides validation as to the mechanism of action of the compound in the assay. Knowledge of the binding mode of fragments is also key to the rapid development of hits that are typically in the micromolar to millimolar potency range. Structure alone, however, tells us nothing about the binding affinity, making it difficult to assess and rank the effectiveness of each fragment hit for further modification. The order in which the methodologies are applied greatly impacts on the size and nature of the fragment library. Fragment libraries screened using X-ray crystallographic methods are at the lower limits, whereas libraries for biochemical screening can be significantly larger in terms of both molecular size and library numbers. Indeed, at the upper limits of what can be construed as fragment screening, Plexxikon ${ }^{[25]}$ use a high-concentration biochemical assay to identify compounds from a library of $\sim 20000$ scaffolds. Scaffolds are noted to be smaller, less potent and less complex than traditional HTS compounds but with MWs up to 350 Da they are obviously larger than the aforementioned library compounds. Hits from the high-concentration biochemical assay are validated using X-ray crystallography, an approach that has found favour in many companies, especially those familiar with crystallography as a tool in drug development.

The definitions described above, although more restrictive than drug-like criteria, still encompass a broad range of molecules, for example optimisation of a $1 \mathrm{mM}$ inhibitor with MW $150 \mathrm{Da}$ is a far better prospect than optimising a $1 \mathrm{mM}$ inhibitor with MW $300 \mathrm{Da}$. Can you quantify the quality of a fragment hit in terms of its potential for transformation into a drug-like molecule? Hadjuk ${ }^{[26]}$ attempted to rationalise the selection of fragments for initiating discovery programmes through a retrospective analysis of the development of a number 
of highly potent inhibitors. By tracing back the end compounds through to constituent fragments and analysing the change in physicochemical properties with potency, he formulated a measure of the likelihood of developing a drug-like molecule from a particular hit fragment based on the size and potency of that molecule. Essentially, potency was observed to increase proportionately with mass along an ideal optimisation path, suggesting that ligand efficiency, discussed further below, should be used in the process of both selecting the most desirable hit fragments and also evaluating the effectiveness of each modification in the development phase. Hadjuk's analysis looks at requirements for individual fragment hits; Makara ${ }^{[27]}$ investigated another aspect of fragment screening, library size, in particular in relation to sampling available fragment space. Key conclusions are that a relatively small number of fragments yield hits against many targets with libraries of $10^{3}$ compounds providing more than sufficient chemical matter to follow up. Diversity, in this instance across the reagents used to construct the library, is vital in formulating a fragment screening deck.

What, then, is a fragment? As for drug-like classification, there is no single, unifying definition that can categorically distinguish between fragments and non-fragments, but there are some general rules of thumb:

- Fragments are smaller than drug-like molecules, whether that is defined by MW or the number of heavy atoms.

- Fragments, in addition to being smaller, are less complex than drug-like molecules.

- Fragments, due to the manner in which they are used, should be highly soluble.

- Fragments should be devoid of undesirable chemical functionality while at the same time facilitating rapid development to more potent compounds.

\subsection{Why Use Fragments?}

There are a multitude of reasons for the current popularity of using fragments in drug discovery stemming from both the conceptual advantages of using fragments and the observed failures of alternative methodologies. As stated above, the concept of using fragments arose in the early 1980s when it was theorized that the total affinity of a molecule could be taken as a function of the affinity of constituent components. However, it was only in the mid1990s, with technological advances in the ability to detect weakly binding fragments, that theory was put into practice with what is often cited as the first demonstration of fragmentbased drug discovery. ${ }^{[28]}$ In the meantime, drug discovery became a game of numbers as it was perceived that quantity could compensate for a lack of understanding with the development of HTS and combinatorial chemistry. HTS has had successes and is still the predominant means of hit identification, but it was quickly acknowledged that screening large compound libraries has many shortcomings and if anything led to higher attrition rates. Subsequently, screening libraries were overhauled based on drug/lead-like characteristics, high-content screening was introduced and ultimately companies looked to alternative means of initiating discovery programmes including ever more sophisticated fragment based approaches.

One of the postulates of fragment-based drug design is that considerable gains in potency can be achieved through linking together fragments binding in different regions of the 
protein site due to free energy considerations in the shift to a single entity. Indeed, initial attempts to exploit fragment-based drug design centred on the strategy of linking fragments; the ideas behind the strategy have since been explored further. Murray and Verdonk ${ }^{[29]}$ analysed the change in the free energy of binding for a molecule formed through linking two separate fragments. Essentially the total free energy of binding for a fragment is divided into two components, the free energy associated with the loss of rigid body entropy and the remaining free energy contribution, termed the intrinsic free energy, which incorporates factors such as the protein-ligand interactions and intramolecular conformational restriction of the fragment. The free energy for the fragment-linked molecule is then a summation of both fragment intrinsic free energies, additional free energy terms associated with the linking group and only a single rigid body free energy contribution. The magnitude of the rigid body term is estimated to be $15-20 \mathrm{~kJ} \mathrm{~mol}^{-1}$ and independent of the size of the molecules under consideration. The larger fragment linked molecule only then incurs the same entropic penalty as for a smaller fragment, thus leading to a saving in the total entropic penalty as compared with the individual fragments. Fragment linking has shown limited success as it is very much dependent on the ability to link chemically the individual fragments without significantly perturbing the effectiveness of the fragment receptor interactions. As discussed previously, this is by no means easy and careful consideration must be taken in the design of the fragments and the ability to link the fragments synthetically in the context of the receptor. It is also the case that it is less common to identify fragments that could bind simultaneously in adjacent regions of the target binding site. It may be simply because there is insufficient space in the binding site to recognize multiple fragments simultaneously or adjacent sites, if available, may not provide a suitable environment for fragment recognition. As discussed below, features in a binding site are unlikely to be distributed evenly, meaning that there is a greater probability of identifying fragments that match a specific region in a site. Alternatively, what has proved more amenable is the merger of two fragments into a larger, more potent, compound or more simply the use of the structural information to rationally improve upon a fragment hit (Figure 1.1). There is an increased probability of identifying the separate, less complex fragments compared with the larger, more potent, compound and also a greater chance of achieving maximum affinity.

The effectiveness of the interactions between a receptor and a small molecule is nowadays discussed in terms of ligand efficiency. There are many different ligand efficiency indices, but essentially they are all a means of normalising the affinity of a molecule with respect to the size of that molecule,${ }^{[30]}$ thereby providing a measure of the quality of fit for that molecule to the receptor. Quality of fit is an important consideration in both the selection of compounds from a screen, whether that is a screen of drug-like molecules or a fragmentbased screen, and in the development of molecules through to preclinical candidates. The origins of ligand efficiency can be seen in earlier work investigating ligand-receptor interactions. Our understanding of small molecule-protein interactions is as yet insufficient to be able to predict binding affinities accurately, however, from an analysis of existing data Andrews et al. ${ }^{[31]}$ formulated a means of ranking drug-receptor interactions based on known functional group contributions. Small charged groups were found to contribute significantly, followed by polar groups and finally nonpolar groups. The experimentally observed binding affinity of a molecule can then be compared with an estimated value obtained by summing the intrinsic binding energies for these constituent groups taking into account entropic penalties. If the affinity is greater than average, then the fit to the receptor 


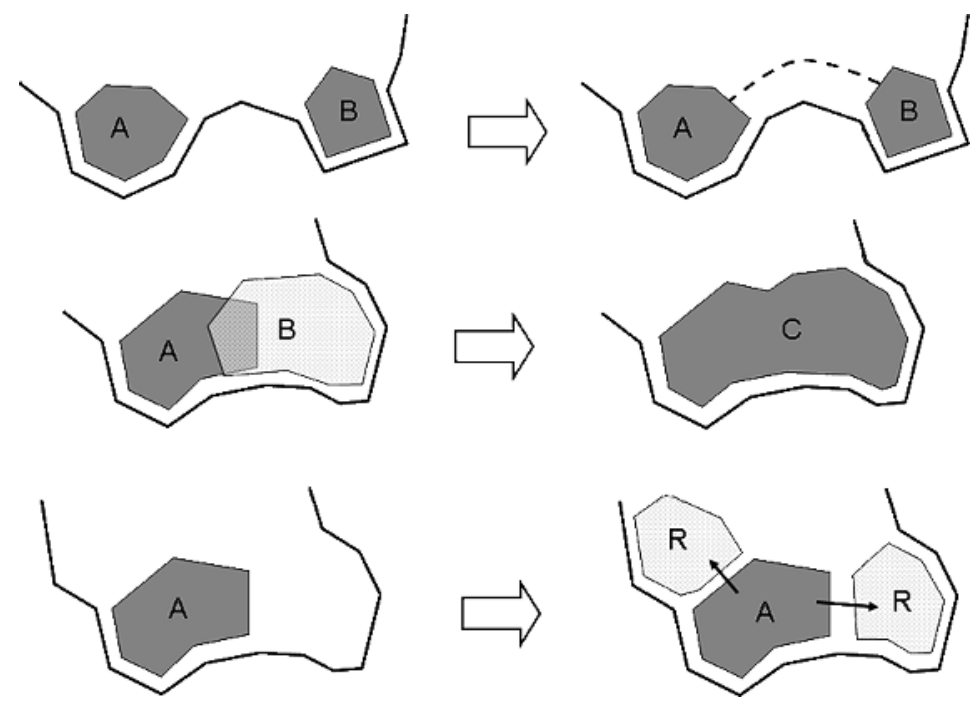

Figure 1.1 Fragment development strategies. Top: fragment linking, where fragments found to bind in adjacent regions of the binding site are linked to create a larger, more potent compound. Middle: fragment fusion, where fragments in overlapping space are amalgamated to form a larger more potent compound. Bottom: fragment growth, where rational design is used to grow the core fragment into adjacent regions of the binding site.

is good, and if not, then it is suboptimal, giving the medicinal chemist a qualitative guide to designing the next round of molecules. Kuntz et al. ${ }^{[32]}$ extended the analysis, looking at the maximum affinity of ligands. Strong binding ligands were taken as references for understanding potential free energy gains as the number of heavy atoms in a molecule is increased. They came to the conclusion that increasing the number of non-hydrogen atoms up to 15 heavy atoms can increase the affinity by $\sim 1.5 \mathrm{kcal} \mathrm{mol}^{-1}$ per atom; beyond that, the free energy was found not to increase linearly with increasing molecular size. Interestingly, van der Waals interactions and hydrophobic effects are now able to explain affinities for most ligands and only in particular cases do atoms such as metal ions dominate binding. The work of Kuntz et al. was instrumental in the use of ligand efficiencies to assess the binding affinity of compounds. With access to superior datasets, subsequent studies suggested that ligand efficiency is dependent on molecular size and does not exhibit the linear relationship below 16 atoms as proposed by Kuntz et al. Reynolds et al. ${ }^{[33]}$ noted that smaller molecules can demonstrate considerably higher ligand efficiencies than observed for larger drug-like molecules. This has important implications when comparing hits from a wide range of molecular sizes; indeed Reynolds et al. propose a size-normalized efficiency scale termed 'fit quality' as a metric for assessing the goodness of fit between ligand and receptor. Essentially, a maximum ligand efficiency is calculated, based on existing data, for each heavy atom count and the ligand efficiency for a particular molecule is scaled according to the optimum curve. Murray and Verdonk ${ }^{[29]}$ also stated that smaller molecules necessitate more optimal binding interactions in order for the intrinsic free energy to surmount the entropic free energy penalty. 
Ligand efficiency provides a means, as discussed by Hadjuk, ${ }^{[26]}$ of extrapolating from a screening hit to determine if a potency objective can be achieved through additions to the molecule while maintaining a desired physicochemical property profile. One of the advantages of using fragments is that they leave more scope for improvement based on a typical medicinal chemistry approach (Figure 1.2), where studies ${ }^{[34]}$ have shown that lead development increases both the size and lipophilicity of the original hits. The fact that existing data suggest fragments exhibit higher ligand efficiencies may also be a consequence of traditional development strategies where suboptimal hits were taken as starting points and/or suboptimal modifications were made in the development process. A clearer picture may well develop as current practices of using ligand efficiency in both the selection and development of compounds feeds into our knowledge base. It is also the case that the different features around a receptor binding site lead to an uneven distribution in the maximum binding affinity across the site. The same can be said for screening hits where different components of a ligand will contribute differently to the total binding affinity. Without additional SAR information, it can be very difficult to establish, even with knowledge of the binding pose, the contribution of each component to the total binding affinity, thereby necessitating the deconstruction of that hit and subsequent testing of the individual components, i.e. fragment-based screening.

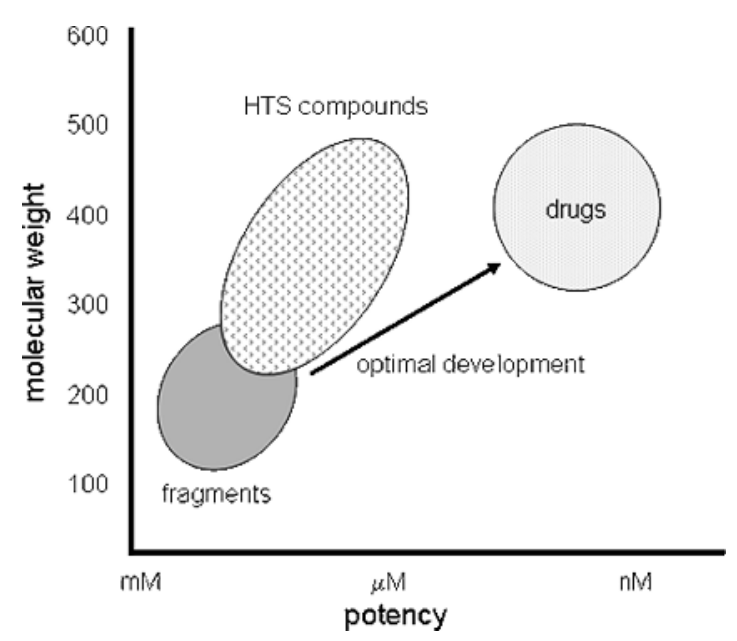

Figure 1.2 Fragments provide greater scope for development into drug-like molecules as compared with HTS molecules that exhibit suboptimal binding to the target protein.

The dynamic, complex nature of receptor binding sites also highlights another very important benefit of the fragment-based approach, that of the probability of identifying an optimal match to the receptor. Hann et al. ${ }^{[22]}$ calculated the probability of a binding event with varying complexity of ligand-receptor interactions and noted that the chance of observing a useful interaction falls rapidly with increasing complexity. The process of binding was reduced to a simple functional model of molecular recognition between the features of the receptor and a ligand. All the standard pharmacophoric features, such as 
donors and acceptors, were represented as $+\mathrm{s}$ and $-\mathrm{s}$ with a positive interaction occurring between $\mathrm{a}+$ on the receptor and $\mathrm{a}-$ on the ligand or vice versa. An exhaustive calculation was then performed, computing the probability of finding an exact match between a receptor of given complexity and a ligand with increasing complexity and, as expected, the probability diminishes rapidly as the number of possible permutations increases. It is also the fact that as one increases the complexity of a molecule the probability of negative interactions increases, leading to suboptimal binding. This has a direct bearing on the size of a screening library, with studies suggesting that even a million compounds in an HTS collection barely scratches the surface of the number of possible molecules in drug-like chemical space ${ }^{[35]}$. Counter to the argument of reducing complexity to a bare minimum in order to maximise the probability of identifying hits, there is a lower limit imposed on the complexity due to the sensitivity of the screening protocol. Hence there is a balance between the probability of there being an exact match between receptor and ligand and the ability to detect that match in a screen. For a particular screening protocol, this leads to an optimum level of ligand complexity, which in turn dictates the size of the screening library necessary to maintain a sufficient hit rate.

Fragment-based drug discovery goes some of the way to compensating for our incomplete understanding of biological interactions and provides a complementary if not alternative route to finding chemical matter for discovery programmes:

- The smaller, less complex nature of fragments increases the probability of finding a match to a receptor; moreover, instances have shown that removing complexity by screening fragments can succeed where HTS has failed [6].

- Fragment libraries can be smaller than HTS libraries as hit rates are generally higher than in traditional HTS due to better sampling and the increased probability of identifying a match to the receptor. This has many advantages associated with the construction, storage and screening of fragment libraries versus HTS libraries.

- Fragment-based screening can also identify more optimal matches (higher ligand efficiency) to the receptor without the need first to deconstruct a hit compound.

- Fragment hits then provide greater scope for development when following a standard medicinal chemistry development strategy (Figure 1.2).

- Screening fragments requires more sensitive detection methods, but at the same time these methods provide invaluable information in the development from hits to drug candidates.

\subsection{Practical Implications of Using Fragments in Drug Discovery}

The primary application of fragments is in the identification of chemical matter to take forward into drug development. As the intention is to identify molecules that are typically in the micromolar to millimolar potency range, a method of detection is required that is more sensitive than a biochemical screen at $10-30 \mu \mathrm{M}$. Assays can be adapted to screen molecules at higher concentrations but, as discussed above and in following chapters on fragment library design, more stringent requirements are placed on the molecules. It is also paramount to obtain confirmation of the mechanism of action as screening at higher concentrations leads to higher false positive rates. Most if not all fragment screening strategies 
will then incorporate a biophysical technique either as the primary screen or to validate hits obtained from an alternative source. NMR and X-ray crystallography are undoubtedly the most popular approaches, as highlighted in ensuing chapters, requiring substantial investment in terms of skill base and technology. Drug discovery programmes, however, benefit immensely from access to structural information at this critical stage, when the chemical nature of the lead compounds is being decided, and thus structural biology can have a significant positive impact on the speed and success of the programme. ${ }^{[36]}$ Effective dissemination of the information gained from a screen is also decisive both in understanding the activity of the hit fragments and in their development. Here computational chemistry and informatics tools can play a key role in integrating data across the different disciplines in addition to directing the design of follow-up compounds. Development can follow one of many strategies, from simple elaboration of single compounds, linking fragments from adjacent regions in a binding site, focused libraries around one or more hits or to more complex amalgamations of compounds observed to bind in overlapping regions of the binding site. Having access to multiple structures of fragment-protein complexes is an invaluable tool in this process; it can also provide a direct understanding of the protein binding site and guide optimisation throughout the lifetime of the project. The final chapters will review the use of computational methods in the overall process and then lead on to practical examples of fragment-based drug discovery.

\section{References}

[1] Leach, A. R., Hann, M. M., Burrows, J. N., and Griffen, E.J. (2006). Fragment screening: an introduction. Mol. BioSyst. 2, 429-446.

[2] Rees, D. C., Congreve, M., Murray, C. W., and Carr, R. (2004). Fragment-based lead discovery. Nat. Rev. Drug Discov. 3, 680-672.

[3] Zartler, E. R., and Shapiro, M. J. (2005). Fragonomics: fragment-based drug discovery. Curr. Opin. Chem. Biol. 9, 366-370.

[4] Erlanson, D. A., McDowell, R. S., and O’Brien, T. (2004). Fragment-based drug discovery. J. Med. Chem. 47(14), 3463-3482.

[5] Mitchell, T., and Cherry, M. (2005). Fragment-based drug design. Innov. Pharm. Technol. 16, 34-36.

[6] Boehm, H. J., Boehringer, M., Bur, D., Gmuender, H., Huber, W., Klaus, W., Kostrewa, D., Kuehne, H., Luebbers, T., Meunier-Keller N., and Mueller, F. (2000). Novel inhibitors of DNA gyrase: 3D structure based biased needle screening, hit validation by biophysical methods and 3D guided optimization. a promising alternative to random screening. J. Med. Chem. 43, 2664-2674.

[7] Lipinski, C.A., Lombardo, F., Dominy, D. W., and Feeney P. J. (1997). Experimental and computational approaches to estimate solubility and permeability in drug discovery and development settings. Adv. Drug Deliv. Rev. 23, 3-25.

[8] Landers, P. (2004). Drug industry's big push into technology falls short: testing machines were built to streamline research - but may be stifling it. Wall Street J. February 24.

[9] Gribbon, P., and Sewing, A. (2005). High-throughput drug discovery: what can we expect from HTS?. Drug Discov. Today 10, 17-22.

[10] Bohacek, R. S., McMartin, C., and Guida, W. C. (1996). The art and practice of structure-based drug design: a molecular modeling perspective. Med. Res. Rev. 16, 3-50. 
[11] Erlanson, D. A., and Jahnke, W. (2006). In Fragment-Based Approaches in Drug Discovery, ed Jahnke, W., and Erlanson, D. A., Wiley-VCH Verlag GmbH, Weinheim, pp. 3-9.

[12] Jennings, A., and Tennant, M. (2005). Discovery strategies in a biopharmaceutical startup: maximising your chances of success using computational filters. Curr. Pharm. Des. 11, 335-344.

[13] Teague, S. J., Davis A. M., Leeson, P. D., and Oprea, T. (1999). The design of leadlike combinatroial libraries. Angew. Chem. 38, 3743-3748.

[14] Congreve, M., Carr, R., Murray, C., and Jhoti, H. (2003). A 'rule of three' for fragment-based lead discovery? Drug Discov. Today 8, 876-877.

[15] Lipinski, C. A., Lombardo, F., Dominy, B. W., and Feeney, P. J. (2001). Experimental and computational approaches to estimate solubility and permeability in drug discovery and development settings. Adv. Drug Deliv. Rev. 46, 3-26.

[16] Baurin, N., Baker, R., Richardson, C., Chen, I., Foloppe, N., Potter, A., Jordan, A., Roughley, S., Parratt, M., Greaney, P., Morley, D., and Hubbard, R. E. (2004). Drug-like annotation and duplicate analysis of a 23 -supplier chemical database totalling 2.7 million compounds. J. Chem. Inf. Comput. Sci. 44, 643-651.

[17] Blaney, J., Nienaber, V., and Burley, S. K. (2006). In Fragment-Based Approaches in Drug Discovery, ed. Jahnke, W. and Erlanson, D.A., Wiley-VCH Verlag GmbH, Weinheim, pp. 215-248.

[18] Fejzo, J., Lepre, C. A., Peng, J. W., Bemis, G. W., Ajay, Murcko, M. A., and Moore, J. M. (1999). The SHAPES strategy: an NMR-based approach for lead generation in drug discovery. Chem. Biol. 6, 755-769.

[19] Bemis, G. W., and Murcko, M. A. (1996). The properties of known drugs. 1. Molecular frameworks. J. Med. Chem. 39, 2887-2893.

[20] Bemis, G. W., and Murcko, M. A. (1999). Properties of known drugs. 2. Side chains. J. Med. Chem. 42, 5095-5099.

[21] Lewell, X. Q., Judd, D. B., Watson, S. P., and Hann, M.M. (1998). RECAP - retrosynthetic combinatorial analysis procedure: a powerful new technique for identifying privileged molecular fragments with useful applications in combinatorial chemistry. J. Chem. Inf. Comput. Sci. 38, 511-522.

[22] Hann, M. M., Leach A. R., and Harper G. (2001). Molecular complexity and its impact on the probability of finding leads for drug discovery. J. Chem. Inf. Comput. Sci. 41, 856-864.

[23] Schuffenhauer, A., Ruedisser, S., Marzinzik, A. L., Jahnke, W., Blommers, M., Selzer, P., and Jacoby, E. (2005). Library design for fragment based screening. Curr. Top. Med. Chem. 5, 751-762.

[24] Baurin, N., Aboul-Ela, F., Barril, X., Davis, B., Drysdale, M., Dymock, B., Finch, H., Fromont, C., Richardson, C., Simmonite, H., and Hubbard, R. E. (2004). Design and characterization of libraries of molecular fragments for use in NMR screening against protein targets. J. Chem. Inf. Comput. Sci. 44, 2157-2166.

[25] Card, G. L., Blasdel, L., England, B. P., Zhang, C., Suzuki, Y., Gillette, S., Fong, D., Ibrahim, P. N., Artis, D. R., Bollag, G., Milburn, M. V., Kim, S.-H., Schlessinger, J., and Zhang, K. Y. J., (2005). A family of phosphodiesterase inhibitors discovered by cocrystallography and scaffoldbased drug design. Nat. Biotechnol. 23, 201-207.

[26] Hadjuk, P. J. (2006). Fragment-based drug design: how big is too big?. J. Med. Chem. 49, 6972-6976.

[27] Makara, G. M. (2007). On sampling of fragment space. J. Med. Chem. 50, 3214-3221.

[28] Shuker, S. B., Hajduk, P. J., Meadows, R. P., and Fesik, S. W. (1996). Discovering high-affinity ligands for proteins: SAR by NMR. Science 274, 1531-1534.

[29] Murray, C., and Verdonk, M. L. (2006). In Fragment-based Approaches in Drug Discovery, ed. Jahnke, W. and Erlanson, D.A., Wiley-VCH Verlag GmbHWeinheim, pp. 55-66. 
[30] Abad-Zapatero, C., and Metz, T. (2005). Ligand efficiency indices as guideposts for drug discovery. Drug Discov. Today 10, 464-469.

[31] Andrews, P. R., Craik, D. J., and Martin, J. L. (1984). Functional group contributions to drug-receptor interactions. J. Med. Chem. 27, 1648-1657.

[32] Kuntz, I. D., Chen, K., Sharp, K. A., and Kollman, P. A. (1999). The maximal affinity of ligands. Proc. Natl. Acad. Sci. USA 96, 9997-10002.

[33] Reynolds, C. H., Bembenek, S. D., and Tounge, B. A. (2007). The role of molecular size in ligand efficiency. Bioorg. Med. Chem. Lett. 17, 4258-4261.

[34] Oprea, T. I., Davis, A. M., Teague, S. J., and Leeson, P. D. (2001). Is there a difference between leads and drugs? A historical perspective. J. Chem. Inf. Comput. Sci. 41, 1308-1315.

[35] Hann, M. M., and Oprea, T. I. (2004). Pursuing the leadlikeness concept in pharmaceutical research. Curr. Opin. Chem. Biol. 8, 255-263.

[36] Stevens, R.C. (2004). Long live structural biology. Nat. Struct. Mol. Biol. 11, 293-295. 
